\title{
Nosocomial Infection Agents of Şişli Hamidiye Etfal Training and Research Hospital: Comparison of 1995 and 2017 Data
}

\section{(1) Mehmet Emin Bulut, ${ }^{1}$ (i) Ahsen Oncul, ${ }^{2}$ (1) Engin Seber ${ }^{2}$}

'Medical Microbiology Laboratory, Health Sciences University Şişli Hamidiye Etfal Training and Research Hospital, Istanbul, Turkey ${ }^{2}$ Department of Infectious Diseases and Clinical Microbiology, Health Sciences University Şişli Hamidiye Etfal Training and Research Hospital, Istanbul, Turkey

\section{Corrigendum}

DOI: 10.14744/SEMB.2019.03271

Med Bull Sisli Etfal Hosp 2020;54(1):78-82

The list of authors was incomplete in the original publication.

The correct list is:

Mehmet Emin Bulut, Ahsen Oncul, Engin Seber 